BESANÇON

Algebraic construction of bilinear forms over $\mathbb{Z}$ 


\title{
Algebraic construction of bilinear forms over $\mathbb{Z}$
}

\author{
Martin Krüskemper
}

\begin{abstract}
Chapter 8 in [CS] gives algebraic constructions for certain lattices. Some of these constructions used the trace map. In this note we want to show that by applying [S],[W], any bilinear form over $\mathbb{Z}$ with nonzero determinant can be constructed as a scaled trace form of some algebra $A=\mathbb{Z}[X] /(f(X))$ where $f(X) \in \mathscr{Z} X X]$ is monic, irreducible.
\end{abstract}

Let $R$ be a commutative ring with 1 . A bilinear form over $R$ is a pair $(M, b)$ where $M$ is a finitely generated projective $R$-module and $b: M \times M \rightarrow R$ is symmetric bilinear. If $M$ is free and $e_{1}, \ldots, e_{n}$ is a basis for $M$ then $l$ can be described by the symmetric $n \times n$ matrix $B=\left(b\left(e_{i}, e_{j}\right)\right)$ over $R$. (Conversely any $B \in S y m(n, R)$ defines the bilinear form $\left(R^{n}, B\right)$. The determinant. de: $l(M, b)$ is defined as $\operatorname{det}(M, b)=\operatorname{det} B .(M, b)$ is called regular if $\operatorname{det}(M, b)$ is an unit in $R$. We call two forms $(M, b)$ and $\left(M^{\prime}, b^{\prime}\right)$ with matrices $B, B^{\prime} \in S y m(n, R)$ isometric if there exists an invertible $M \in M a t(n, R)$ such that $B=M^{t} B^{\prime} M$.

If $\beta: R \rightarrow A$ is a ringmorphism such that $A$ is a finitely generated projective $R-$ module and $s \in H_{0 m}(A, R)$, then the map $(x, y) \in A \times A \rightarrow s(x y)$ defines a bilinear form $(A, s)$ over $R$. More generally, if $\mathcal{I}$ is an ideal in $A$ such that $\mathcal{I}$ is a finitely generated projective $R$-module then $(x, y) \in I \times I \rightarrow s(x y)$ defines a bilinear form $(I, s)$ over $R$. We call $(I, s)$ scaled trace form of $A / R$.

Let $f(X) \in \mathbb{Z}[X]$ be a monic separable polynomial of degree $n$ (in one variable $X$ ). Then $A:=\mathbb{Z}[X] /(f(X))$ is a free $\mathbb{Z}$-mudule of rank $n$ with basis $1, X, \ldots, X^{n-1}$. We set $A_{\mathbb{Q}}:=A \otimes \mathbb{Q}$. If $f$ is irreducible then $A_{\mathbb{Q}}=Q(A)$ denotes the quotient field of $A$. Let $\operatorname{Tr}: A_{\mathbb{Q}} \rightarrow \mathbb{Q}$ clenote the trace map which is non-zero. Euler's lemma (see [L] III-1, proposition 2, corollary) implies that we have:

$$
A^{\#}:=\left\{c \in A_{\mathbb{Q}} \mid \operatorname{Tr}(c A) \subset \mathbb{Z}\right\}=1 /\left(f^{\prime}(X)\right) A .
$$

Any $c \in A^{\#}$ defines a symmetric bilinear form $\left(A, T r_{c}\right)$ where $T r_{c}$ maps $\left(a_{1}, a_{2}\right) \in A \times A$ to $\operatorname{Tr}\left(c\left(u_{1}\left(\alpha_{2}\right)\right.\right.$. Let $N: A_{\mathbb{Q}} \rightarrow \mathbb{Q}$ denote the nurm map. It is well known (compare [L] III-1) that if $c=c_{0} / f^{\prime \prime}(X) \in A^{\#}$, where $c_{0} \in A$, then we have $\operatorname{det}\left(A, T r_{c}\right)=$ $(-1)^{n(n-1) / 2} N\left(c_{u}\right)$. In particular, it $c \neq 0$ then det $\left(A, T r_{c}\right) \neq 0$ and the form $\left(A, T r_{c}\right)$ is regular if $c_{0}$ is a unit in the integial closure. More generally, let $\mathcal{I} \subset A$ be an ideal in $A$ and let $c \in\left(I^{\prime}\right)^{\#}$. Then we obtain a symmetric bilinear form $\left(I, T r_{c}\right)$ if $T r_{c}$ matps $\left(a_{1}, a_{2}\right) \in \mathcal{I} \times \mathcal{I}$ to $\operatorname{Tr}\left(c a_{1}\left(a_{2}\right)\right.$. Note that if $\mathcal{B}$ is an ideal in $A$ then $\mathcal{B}^{\#}=\mathcal{B}^{-1} A^{\#}=1 /\left(f^{\prime}(X)\right) \mathcal{B}^{-1}$. If $c=c_{0} / f^{\prime}(X)$ where $c_{0} \in \mathcal{I}^{-2}$ we have $\operatorname{det}\left(\mathcal{I}, T r_{c}\right)=$ $(-1)^{n(n-1) / 2} N\left(c_{0}\right) N(\mathcal{I})^{2}$. (Compance [CS ] pange 226.)

An obvious question is the lollowing: Let $(M, b)$ be a scaled trace form of $A / Z \mathbb{Z}$. If $\left(N, b^{\prime}\right)$ is symmetric bilinear such that $(M, b) \bigcirc \mathbb{Q}$ is isometric to $\left(N, b^{\prime}\right) \otimes \mathbb{Q}$, then is 
$\left(N, b^{\prime}\right)$ also a scaled trace form of $A / Z \mathbb{Z}$ ! The following examples show, that the answer to this question is negative:

Examples. (a) Let $d \in \mathbb{Z}$ be not a square and set $A=\mathscr{Z}[\sqrt{d}]$. Let $(M, b)$ be a two-dimensional symmetric bilinear form over $\mathbb{Z}$. Then there exists some $c \in A^{\#}$ such that $(M, b)=\left(A, T r_{c}\right)$ if and only if there exists a $\mathbb{Z}$-basis $m_{1}, m_{2}$ of $M$ such that the matrix of $b$ with respect to $m_{1}, m_{2}$ is in

$$
\left\{\left(\begin{array}{cc}
a & b \\
b & a d
\end{array}\right) \mid a, b \in \mathscr{Z}, a \neq 0 \text { or } \quad b \neq 0\right\}
$$

Proof. Set $\tau:=\frac{a \sqrt{d}+b}{2 \sqrt{d}}$. We obtain as matrix of $\left(A, T r_{\tau}\right)$ with respect to the $\mathbb{Z}$-basis $1, \sqrt{d}$,

$$
\left(\begin{array}{cc}
a & b \\
b & a d
\end{array}\right) .
$$

(b) We consider the field extension $A=\mathbb{Q}[\sqrt{d}] / \mathbb{Q}$. It is easy to chek that a twodimensional symmetric bilinear form $(M, b)$ over $\mathbb{Q}$ is scaled trace form of $A / \mathbb{Q}$ if and only if $0 \neq-\operatorname{det}(M, b)$ is a norm.

(c) Let $A=\mathscr{Z}[\sqrt{-1}]$. Since $A$ is a principal icleal domain, example (a) describes all scaled trace forms of $A / \mathbb{Z}$ : A two-dimensional form of determinant -4 is a scaled trace form of $A / \mathbb{Z}$ if and only if it is given by a matrix of the followig type

$$
\left\{\left(\begin{array}{cc}
a & b \\
b & -a
\end{array}\right) \mid a= \pm 2, b=0 \text { or } a=0, b= \pm 2\right\} \text {. }
$$

These matrices describe only two different isometry classes of forms over $\mathbb{Z}$. It is well known that there exists more than two liflerent isometry classes of symmetric bilinear forms over $\mathbb{Z}$ with determinant -4 (S)e [CS] page 3(22.); more precisely, the form given by the matrix

$$
\left(\begin{array}{ll}
0 & 2 \\
2 & 1
\end{array}\right)
$$

is not a scaled trace form of $A / Z$. Since over $Q$ there exists only one isometry class of forms with determinant -4 we see that the answer to the above question is negative.

The following problem remains open: Let $l \in \mathbb{Z}$ be not a square and $A=\mathscr{Z}[\sqrt{d}]$. Then determine all isometry classes of scaled trace forms of $A / \mathbb{Z}$. Let $D \in M a t(n, \mathbb{Z})$. Let $\chi_{D}(X)=\operatorname{det}\left(X E_{n}-D\right) \in \mathscr{Z}[X]$ be the characteristic polynomial of $D$. The next lemna was shown in [T]. Other proots and generalisations of this result can be found in $[\mathrm{CP}],[\mathrm{IS}],[\mathrm{W}]$.

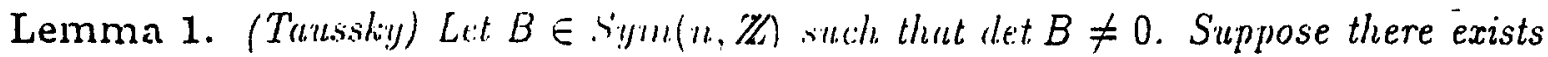
some $M \in M\left(n,(n, \mathbb{Z})\right.$ such that $B M M^{\prime}=M B$ and $x_{M}(X)$ is irreducible. Let $A:=$ 
$\mathbb{Z}[X] /\left(X_{M}(X)\right)$. Then the the tists some $c \in A_{\mathbb{Q}}$ und $b_{1}, \ldots, b_{n} \in A$ such that $\mathbb{Z}_{1}+$ $\ldots+\mathbb{Z b}_{n}$ is an ideal in $A$ and $B=\left(T r\left(c b_{i} b_{j}\right)\right)$. Furthermore $\left(b_{1}, \ldots, b_{n}\right) \in Q(A)^{n}$ is an eigenvector of $M \in M a t(n, Q(A))$.

Note that the above $c$ is in $\left(\mathbb{Z} b_{1}+\ldots+\mathbb{Z} b_{n}\right) \#$. Given some $B \in S y m(n, \mathbb{Z})$ we can always find some $M$ such that $B M^{t}=M B$ : Choose any $S \in \operatorname{Sym}(n, \mathbb{Z})$ and set $M:=B S$. Then $B M^{t}=B(S B)=M B($ Compare $([\mathrm{CP}])$.

Lemma 2. For uny $B \in S y m(n, \mathbb{Z})$, det $B \neq 0$ there exists $M \in \operatorname{Mat}(n, \mathbb{Z})$ such that $B M^{t}=M B$ and $\chi M(X) \in \mathbb{Z}[X]$ is irrelucible. Furthermore we may assume that $\chi_{M}(X)$ is totally real, that is all zeros of $\chi_{M}(X)$ are real.

Proof. Let $N=\left(X_{i j}\right)$ be the symmetric $n \times n$ matrix where the coefficients $X_{i j}=X_{j i}$ are new indeterminates. Clioose $C \in G L(n, \mathbb{Q})$ such that $C^{t} B C$ is a diagonal matrix. Since we may view $C^{-1} N\left(C^{-1}\right)^{t}$ as a symmetric matrix with independent indeterminates as coefficients, by [S] or [W] the characteristic polynomial of $\left(C^{t} B C\right) C^{-1} N\left(C^{-1}\right)^{t}$ is irreducible and it is also the chanacteristic polynomial of $B N$. By Hilbert's irreducibility theorem, there exist $a_{i j}=a_{j i} \in(1)$ such that $\chi B\left(a_{i j}\right)(X)$ is irreducible. If we choose $a \in \mathbb{Z}$ such that all $a u_{i j} \in \mathbb{Z}$, then $\backslash B\left(a u_{i j}\right)(X)=a^{n} X B\left(a_{i j}\right)\left(a^{-1} X\right)$ is irreducible. Hence we set $M:=B\left(u a_{i j}\right)$. We liave $B M^{t}=B\left(u a_{i j}\right)^{t} B^{t}=M B$.

By [S], we may choose the $a_{i j}$ ahove, such that $\chi_{B\left(a_{i j}\right)}(X)$ is totally real. But then $\chi_{B\left(u u_{i}, j\right.}(X)$ is totally real as well.

Using the above notations we have shown:

Theorem. Let $(M, b)$ be a biline:ar form oner $\mathbb{Z}$ such that det $(M, b) \neq 0$. Then there exist a monic, irreducible $f(X) \in \mathbb{Z}[X]$, some ideal $\mathcal{I} \subset A:=\mathbb{Z}[X] /(f(X))$, and $c \in\left(\mathcal{I}^{2}\right)^{\#}$ such that $(M, b)=\left(\mathcal{I}, T r_{c}\right)$. We may ussume thut $f(X)$ is totally real.

Remarks. (a) The result holds more generally for $(M, b)$ over $R, \operatorname{det}(M, b) \neq 0$ where $R$ is the integral closure of $\mathbb{Z}$ in some finite field extension $F / \mathbb{Q}$ and $M$ is finitely generated free. We can also choose $R=k[X]$ where $k$ is a field.

(b) Let $B \in S^{\prime} y m(n, \mathbb{Z})$ such that det $B \neq 0$ and there exists some $c \in A_{\mathbb{Q}}$ and $b_{1}, \ldots, b_{n} \in A$ such that $B=\left(\operatorname{Tr}\left(c b_{i} b_{j}\right)\right)$. Let $B^{\prime} \in \operatorname{Sym}(n, \mathbb{Z})$ such that there exists $C \in M a t(n, \mathscr{Z})$ with $\operatorname{det} C= \pm 1$ and $B^{\prime}=C^{t} B C$. Then there exists some $c^{\prime} \in A_{\mathbb{Q}}$ and $a_{1}, \ldots, a_{n} \in A$ such that $B^{\prime}=\left(\operatorname{Tr}\left(c^{\prime} a_{i}\left(a_{j}\right)\right)\right.$. Furthermore, for the ideals we have $\mathbb{Z}_{1}+\ldots+\mathbb{Z}_{u_{n}}=\mathbb{Z} u_{1}+\ldots+\mathbb{Z} u_{t_{n}}$.

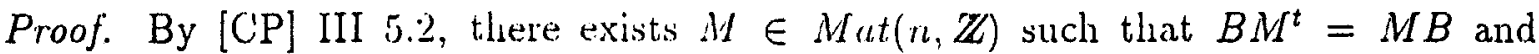
$A=\mathbb{Z}[X] /\left(\chi_{M}(X)\right)$. Then

$$
\left(C^{t} M\left(C^{t}\right)^{-1}\right)\left(C^{t} B C\right)=C^{t} B M^{t} C=\left(C^{t} B C\right)\left(C^{t} M\left(C^{t}\right)^{-1}\right)^{t}
$$


Question. Let $(M, b)$ be a bilinear form over $R$, where $R$ is the integral closure of $\mathbb{Z}$ in some finite field extension $F / \mathbb{Q}$, such that $M$ is not a free but a projective $R$-module. Can we realize $(M L, b)$ as scaled trace lom of some $R$-algebra $A$ ?

\section{REFERENCES}

[CP] Conner, P. E. , Perlis, R. , A survey of trace forms of algebraic number fields. Workd Scientific, Singapore, 1984.

[CS] Conway, J. H. , Sloane, N. J. A . Sphere packings, lattices and groups. Berlin, Heidelberg, New York, Springer 1988.

[IS] Ischebeck, F., Scharlitu, W., Hermitesche und orthogonale Operatoren über kommutativen Ringen. Mathl. An11. 200), 327-334 (1970).

[L] Lang, S., Algebraic muinber theory. Reacling, Massachusetts, Addison Wesley 1970.

[S] Scharlau, W. , On trace forms of algeblatic number fields. Math. Z. 196, 125-127 (1987).

[T] Taussky, O. , On the similarity trans[ormation between an integral matrix with irreducible characteristic polynomial and its transpose. Math. Ann. 166, 60-63 (1966).

[W] Waterhouse, W. C. , Scaled trace lorms over number fields. Arch. Math. 47, $229-231(1986)$.

\section{February 1997}

Mathematisches Institut der Universitït

Einsteinstraße 62

D-48149 Münster

e-mail-adress: kruskem@inath.uni-mueuster.de 